\title{
Mixité et Éducation Physique et Sportive
} (1959-1975)

Les résistances de l'école

Coeducation and physical education (1959-1975): the school resists

Michaël Attali, Cécile Ottogalli-Mazzacavallo et Jean Saint-Martin

\section{CpenEdition}

\section{Journals}

Édition électronique

URL : https://journals.openedition.org/clio/8852

DOI : 10.4000/clio.8852

ISSN : $1777-5299$

Éditeur

Belin

Édition imprimée

Date de publication : 15 décembre 2008

Pagination : 243-260

ISSN : 1252-7017

Référence électronique

Michaël Attali, Cécile Ottogalli-Mazzacavallo et Jean Saint-Martin, « Mixité et Éducation Physique et Sportive (1959-1975) », Clio. Histoire, femmes et sociétés [En ligne], 28 | 2008, mis en ligne le 15 décembre 2011, consulté le 22 avril 2022. URL : http://journals.openedition.org/clio/8852 ; DOI https://doi.org/10.4000/clio.8852 


\title{
Mixité et Éducation Physique et Sportive (1959-1975) Les résistances de l'école
}

\author{
Michaël ATTALI \\ Cécile OtTogalli-MaZZACAVALLO \\ Jean SAINT-MARTIN
}

Entre 1959, où l'école connaît une transformation structurelle majeure par l'intermédiaire de la réforme initiée par Jean Berthoin, et 1975, où le collège unique est instauré sous l'effet des lois Haby, se développe une vaste politique de démocratisation de l'enseignement, unanimement sous-tendue par le concept d'égalité des chances. Dans le même temps, la mixité dans l'enseignement s'installe progressivement. Cette nouvelle organisation pédagogique est-elle l'occasion de promouvoir ou de revendiquer l'égalité entre les sexes?

L'utopie égalitaire de l'école est aujourd'hui amplement contredite et il apparaît évident que la mixité n'y suffit pas ${ }^{1}$. Celle-ci s'impose en effet non seulement sous le poids «d'arguments économiques qui sont au fondement des politiques de l'État » $»^{2}$ mais aussi et surtout sur la base d'une vision sexuée des rôles sociaux. À l'aube de la Cinquième République, mixité et égalité ne sont pas interpellées dans leur éventuelle interaction. L'analyse d'une des principales revues éducatives, l'Éducation Nationale $(E N)^{3}$, révèle qu'il « est fort difficile de penser l'égalité des sexes autrement que par la différence »" Malgré des

La mixité menacée ?, Les rapports du Sénat 2003, n²63 (2003-2004).

Zancarini-Fournel $2004: 32$.

Organe du ministère de l'Éducation nationale à sa création en 1945, cette revue devient deux ans plus tard une revue d'informations susceptibles d'intéresser les membres de l'enseignement public. L'EN est un lieu d'échange visant à impulser une dynamique de rénovation du système éducatif.

4 Rogers $2004: 113$. 
discours universalisants sur la démocratisation à engager, malgré l'omniprésence d'une volonté de réduire les inégalités sociales et culturelles entre les élèves, les discours et les pratiques valorisent l'existence d'aptitudes innées et conçoivent un enseignement adapté aux différences entre les élèves. La mixité, qui aurait pu constituer un volet important des réflexions engagées n'est jamais discutée ou justifiée.

Cette absence de débat interroge. Le sujet est-il tabou, négligeable ou ce désintérêt signale-t-il une évolution non maittrisée ? Ce silence est d'autant plus surprenant qu'avec la «démographisation » du système scolaire, entreprise durant les années soixante, la scolarisation des jeunes filles est «manifestement de plus grande ampleur que celle des jeunes gens pour le secondaire $»^{5}$. Lorsque la mixité s'impose dans les disciplines générales, elle repose sur la philosophie dominante d'une construction inégalitaire des identités sexuées et sur la division «naturelle» des sexes. Il s'agit alors de situer les argumentations développées afin de justifier une différenciation qui fait de la mixité un artefact éducatif formel. Certaines disciplines comme l'éducation physique et sportive (EPS) parviennent même à pousser cette logique de différenciation jusqu'à refuser de mettre en œuvre concrètement la mixité. Mettant en jeu le corps des élèves, cette discipline constitue un effet de loupe des tensions du système éducatif autour de la question de l'égalité des chances. Dès lors, la mixité, entre 1959 et 1975, demeure une contrainte, voire un danger mais aucunement un objectif éducatif.

\section{Le genre des aptitudes : différences et différenciation}

L'exposé des motifs du décret n59-57 du 6 janvier 1959 signé par Jean Berthoin met en exergue la volonté de permettre à tous les élèves d'accéder au savoir en fonction des exigences scolaires et de leur adéquation avec les besoins économiques. L'absence de réflexion sociologique sur ce sujet propulse la notion d'aptitude comme régulateur quasi-exclusif de l'organisation de l'enseignement.

5 Lelièvre \& Lelièvre $1991: 208$. 
L'omniprésence de cette notion ${ }^{6}$ revient à éterniser les conduites, à les marquer du sceau de l'irréversibilité au lieu de les appréhender dans une dynamique où interagissent plusieurs éléments. Ainsi, il existerait des intelligences inégales dépendantes d'une hérédité sous-tendue par des représentations naturalistes. Dans ce cadre, l'école a pour mission de détecter ces aptitudes «acquises naturellement» afin d'adapter l'orientation des élèves aux besoins de l'économie. Si le décret Berthoin constitue en quelque sorte le cadre théorique d'une nouvelle école, la revue EN s'atèle à en justifier les principes en faisant intervenir des spécialistes scientifiques reconnus et en proposant des moyens de sa mise en œuvre effective. Psychopédagogues, psychologues, médecins, philosophes, inspecteurs précisent une notion dont la clarté n'est qu'apparente mais dont l'opérationnalisation doit être rapide. La plupart du temps, l'existence de «dons» explique les différences constatées entre les élèves. Cette question des aptitudes prend une ampleur nouvelle lorsqu'elle est associée au sexe des élèves. Pour beaucoup, l'égalité passe par une prise en compte des particularismes. En matière de différence entre les sexes, les évidences deviennent tellement fortes que souvent l'implicite suffit :

[...] considérons la manière dont on use actuellement des aptitudes. Si on voulait vraiment en tenir compte, il faudrait d'abord distinguer sur bien des points l'éducation des deux sexes, car ceux-ci, sans doute comparables en intelligence générale, diffèrent lorsqu'il s'agit des facteurs de vocabulaire ou de visualisation spatiale [...]. Or, il est remarquable que, au moment même où l'on parle le plus d'école sur mesure, on parle aussi de plus en plus - et ce sont les mêmes voix - d'unifier les enseignements des garçons et des filles ! ${ }^{7}$

Ainsi, au moment même où s'établit la mixité, il est encore difficile de pouvoir proposer une éducation identique aux filles et aux garçons. Pour certains, la justice scolaire et sociale voudrait davantage que les filles et les garçons soient traités de façon différente, d'autant plus que les conditions dans lesquelles s'instaure la mixité laissent à penser que

6 Robert $1993: 43$

7 Château $1960: 3$. 
ce régime est inéquitable en particulier pour les filles. C'est donc bien à partir d'un principe égalitaire que se justifie une inégalité pédagogique.

Dans l'EN, un seul article, celui de l'inspectrice générale de l'Education Nationale, Odette Georges Brunschwig, relate les conséquences de la mixité. Si cette dernière s'impose dans les disciplines intellectuelles, son efficacité n'en est pas moins contestée. La subordination du personnel féminin, le manque d'autorité des enseignantes sur les élèves masculins marquent encore les mentalités et les pratiques enseignantes du début des années 1960. Même si les incidents quotidiens de la vie scolaire ou les réticences des familles forcent l'attention «des éducateurs responsables $»^{8}$, la déception de l'auteure par rapport aux conditions de mise en place et aux effets escomptés de la mixité est manifeste. Pour autant, les évidences d'une pédagogie de la mixité qui respecte les différences naturelles entre les sexes s'imposent comme fondement de la justice scolaire :

Mieux vaut par exemple ne pas trop laisser les filles emprunter aux garçons des jeux, des habitudes scolaires, qui ne leurs conviennent pas toujours. Il n'est pas mauvais de viriliser un peu tous les enfants, et la sentimentalité est aussi nuisible que sotte : elle est d'ailleurs en régression. Mais que certaines fillettes supportent mal l'atmosphère de classes de garçons où rien n'est tenté pour les acclimater, ce n'est pas forcément caprices ou pusillanimité. Il est permis aux futures femmes de souhaiter calme et douceur... tout comme Chrysale !

Cette volonté de promouvoir une éducation spécifique en conformité avec les règles de la nature est régulièrement exprimée dans les articles de l'EN. La recension de l'ouvrage de Jeanne Burniaux en 1965 sur L'éducation des jeunes filles ${ }^{10}$ ou plus tard les propos du docteur G. Mauco rappellent que les femmes doivent rester féminines, c'est-àdire respectueuses du modèle de la mère et de l'épouse largement réinvesti après la Seconde Guerre mondiale. Les modèles identitaires de référence sont parfaitement genrés: «Ce sont des images d'hommes forts et calmes, de femmes épanouies dans leur féminité,

B Bunschwig 1961:5.

Brunschwig 1961: 6 .

10 Le Lay $1965: 5$. 
qui imposent par leur exemple la loi sociale $»^{11}$. Nul ne peut en discuter la validité puisque cette loi sociale se fonde sur des principes « naturels » immanents. L'école doit alors renforcer la prégnance de ces représentations, et ce malgré l'instauration d'une mixité des programmes et des espaces dans les disciplines d'enseignement général.

L'organisation non-mixte de l'Éducation Physique et Sportive offre un effet de loupe des résistances de l'institution scolaire française vis-àvis d'une nouvelle conception de l'égalité entre les sexes. Après 1959 se pérennise une EPS à deux sexes. Lorsque l'EN propose des comparaisons entre l'EPS des filles et des garçons, celles-ci sont parfois dépréciatives pour les premières, mais toujours l'occasion de mettre en valeur les méfaits d'une égalité qui ne pourrait correspondre aux réalités biologiques et culturelles de chacun des sexes. Les productions et les performances corporelles attendues et réalisées par les élèves sont perçues comme le résultat de différences biologiques, d'aptitudes différenciées, irrémédiables, validant ainsi les argumentations utilisées au niveau du système éducatif. En référence à des destinées corporelles visiblement dissemblables, les enseignants d'EPS mettent en scène une sexuation des apprentissages.

La plupart des arguments développés sur le sujet sont présentés sous l'angle du bon sens, de l'observation empirique que nul ne peut discuter car communément évidente. Par le corps doivent se construire et s'exprimer des modèles identitaires de référence liés aux contingences naturalistes. Aux femmes, un corps féminin; aux hommes, un corps masculin ${ }^{12}$. En 1964, l'EN, dans un article présentant « une expérience de gymnastique volontaire » confirme cette prégnance d'un corps sexué dans les représentations sociales des enseignants d'EPS et de leurs inspecteurs généraux. Jean Guilhem insiste sur les témoignages de ces jeunes femmes qui considèrent l'exercice comme «le meilleur moyen d'entretenir mon corps, donc notre beauté $»^{13}$. Les épreuves d'EPS lors des examens, et plus

11 Mauco $1969: 7$.

12 Adler $1960: 20$.

13 Guilhem $1964: 8$. 
particulièrement au baccalauréat, en mettant en place des appréciations distinctes, créent une situation unique à l'école et révèlent l'organisation genrée de la pratique physique scolaire et l'établissement de deux espaces de pratiques caractérisés par leur contenu, leur norme, leur code et leur évaluation ${ }^{14}$. L'EPS contribue alors à renforcer les aptitudes perçues comme innées ${ }^{15}$. Les exercices de garçons laissent une place plus importante à l'acrobatie fondée en particulier sur les déséquilibres et les rétablissements. Les filles doivent proposer des enchaînements basés sur des exercices de maintien, de tenue limitant la mobilité mais insistant sur l'harmonie. La danse, spécifique aux seules pratiquantes, témoigne du poids des représentations sociales genrées.

Outre l'évidence d'une corporéité différenciée entre les sexes, la sexuation de l'enseignement de l'EPS est renforcée par celle des pratiques sportives promues alors support exclusif de son enseignement ${ }^{16}$. Marquées du saut de la masculinité dès leurs origines, les pratiques sportives se sont féminisées au prix de multiples luttes ou adaptations quant à leurs modalités de pratique et à leurs finalités ${ }^{17}$. En athlétisme, en hand-ball comme en gymnastique, les femmes sont acceptées à condition de se plier aux règles d'une mise en jeu adaptée aux spécificités de leur sexe : la modération et l'esthétique. Médecins du sport et pédagogues s'accordent à penser que «l'adresse, la grâce et la souplesse, qualités féminines par excellence devront être les buts recherchés dans une juste mesure par le sport féminin $»^{18}$. Phénomène social et culturel en pleine expansion, le sport active toujours craintes et angoisses lorsqu'il se conjugue au féminin. A l'occasion des Jeux olympiques de Rome en 1960, le sport féminin de compétition est sur

14 Attali \& Saint-Martin 2005 : 207-226.

15 Pour Colette Chaussonnet (1960), le principal problème de l'EPS est celui des différences sexuées des élèves.

16 Attali \& Saint Martin 2004

17 Terret et al. 2005.

18 Encausse Dr 1962 : 214. 
la sellette. Dans l'EN, Roger Bouzinac ${ }^{19}$ met en exergue les conséquences d'une pratique inconsidérée altérant les critères sociaux de la féminité :

Dans tout cet échantillonnage de médailles glanées par les grandes puissances sportives du monde, nous pouvons abandonner le plus grand nombre sans aucune amertume: celles qui ont été enlevées par ces femmes pachydermes, ces dames-canons ou ces moitiés d'hommes (que Florence Pétry-Amiel garde son charme, Wilma Rudolph sa ligne pure et Yolance Balas son record!), toutes celles qui ont été arrachées par des phénomènes, sujets de laboratoire et d'expériences promis à un tragique déracinement le jour où ils auront abandonné les stades et les piscines [...] Nous répugnons tous à voir façonner la jeunesse selon un enseignement qui ne souffrirait aucune discordance et qui commencerait à l'âge le plus tendre pour ne plus lâcher sa proie ${ }^{20}$.

Le sport féminin de compétition agit donc comme un contremodèle scolaire. La nature a fait la femme qui ne peut être dévoyée sous des prétextes culturels. Inspiré par le modèle compétitif du haut niveau, l'enseignement de l'EP est empreint d'une masculinité qui laisse à la marge une grande partie des élèves. Les valeurs, les vertus, les qualités développées par les sports renvoient aux exigences de la masculinité de cette période :

Le goût de l'effort, l'oubli de soi-même, la camaraderie et l'amitié étendus à tous les hommes sont les points essentiels du message qu'il nous a laissé. Ce message est plus que jamais actuel [...] Les jeunes français et en particulier les jeunes sportifs [...] auront à cœur de faire prévaloir cet idéal. Partout et toujours, à l'usine et dans les champs, à l'université et sur les stades, ils seront ainsi les témoins de la vitalité et du rayonnement de la France ${ }^{21}$.

L’idéologie politique en vigueur à cette période rejoint ainsi celle de l'école dans l'application de laquelle l'EPS prend une part considérable. Le corps et l'esprit de l'homme se régénèrent dans un idéal conforme

19 Membre des cabinets ministériels pendant la IVe République, Roger Bouzinac, journaliste devient dans les années 1970, directeur du syndicat national de la presse régionale quotidienne.

20 Bouzinac $1960: 16$.

21 Herzog $1963: 2$. 
et structurant du genre. Le sport proposé en EPS est ainsi considéré comme une pratique d'assignation sociale. En raison des formes de pratique privilégiées (la compétition) et du public visé (les hommes), il constitue à lui seul une école des identités sexuées.

$\mathrm{Au}$ risque de dénaturation du caractère de chaque sexe en efféminant les hommes et en virilisant les femmes, s'adjoint celui de la perversion morale des élèves et de la mise en danger de la virginité des filles, dû à la promiscuité sexuelle que favorise la mixité. En EPS, ces angoisses sont exacerbées par la mise en jeu des corps qui sont avant tout sexués et donc sexuels. Certaines précautions sont ainsi à prendre pour éviter les dangers moraux de la mixité, dont celle de «s'abstenir des jeux corporels et épreuves sportives mixtes $»^{22}$.

Si l'enseignement général compose, dans la première moitié des années 1960, avec une mixité imposée, l'EPS se conforme aux principes d'une éducation séparée et différenciée entre les sexes. Pour répondre aux destinées corporelles souhaitées et pour minimiser les angoisses moralistes, l'enseignement séparé entre les filles et les garçons se pérennise. Loin d'être «en retard» sur les évolutions pédagogiques de son temps, l'EPS est donc en cohérence avec les stéréotypes de la naturalité des différences motrices et sportives et constitue une puissante caisse de résonance des positions éducatives tenues par les divers protagonistes du débat scolaire.

\section{L'utopie d'une contestation au mitan des années 1960}

Autour de la fracture sociale et culturelle des années soixante-huit s'initient des réflexions sur la place et le rôle des femmes dans la société qui rencontrent peu d'écho dans la revue EN. L'engagement de certains pédagogues "féministes», tentant de dénoncer le conservatisme de l'école dans l'éducation des identités sexuées, est extrêmement rare et proche de l'idéologie dominante. La question des enjeux de la mixité n'est pas plus abordée alors que, depuis 1965, se multiplient les études de satisfaction, anglaises ${ }^{23}$, belges ${ }^{24}$ ou franco-

2 Courtois $1967: 46$

23 Dale 1965. 
belge ${ }^{25}$ à l'égard de la mixité. Au mieux, on note en décembre 1970, l'article de Pierre Ferran ${ }^{26}$ qui tente de sensibiliser le lectorat de l'EN sur les avantages pédagogiques de la mixité. Reprenant les conclusions de l'inspecteur général Edouard Breuse en 1970 dans La coéducation dans les écoles mixtes, il estime urgent d'institutionnaliser une véritable coéducation dans le système scolaire, la généralisation de la mixité en étant la première étape.

La coéducation se distingue en effet de la mixité. Elle est «une conception rénovée de l'éducation qui consiste à réunir garçons et filles dans une même école et dans les mêmes classes, et à leur donner ensemble une éducation identique $»^{27}$. Mais surtout contrairement à la mixité, elle "procède nécessairement d'une doctrine qui affirme la valeur spécifique mais complémentaire de chaque sexe et postule la reconnaissance non de leur similitude mais de leur égalité $»^{28}$. Malgré les évolutions sociales et culturelles concernant la place des femmes et les rapports sociaux entre les sexes, l'égalité visée se conçoit toujours exclusivement dans le respect des différences.

Il serait vain, par exemple, de vouloir nier complètement les répercussions des déterminismes biologiques sur le psychisme ; la sensibilité, l'émotivité, la façon générale d'être de la femme dans la vie ${ }^{29}$.

Ainsi, hormis les propositions d'E. Breuse, la généralisation de la mixité ne conduit pas à s'interroger sur un renouvellement des dispositifs pédagogiques. Malgré l'expérience d'une dizaine d'années, les effets de la mixité sur les apprentissages ou la diversité des formes qu'elle peut revêtir ne sont presque jamais évoqués dans la revue EN. Partout, la mixité semble devenue un fait d'évidence, une réalité

24 Bastin 1966.

25 Les cabiers de l'INAS 1965.

26 Ferran 1970. Instituteur et titulaire d'une maitrise de lettres, Pierre Ferran est enseignant-chercheur à l'ENS St-Cloud au cours des années 1960.

27 Breuse $1970: 107$.

28 Breuse $1970: 108$.

29 Breuse $1970: 49$. 
quotidienne que nul ne pense à interroger, à étudier, voire à remettre en question sur le plan de l'égalité des sexes.

Mais, ce fait d'évidence ne concerne cependant pas l'EPS où la non mixité reste la règle dans les enseignements de la seconde moitié des années 1960. Révélatrice des résistances de fond à l'égard d'une nouvelle égalité, l'EPS n'évolue guère pendant ces années de contestation sociale. Malgré l'apparence d'un discours asexué qui devient majoritaire dans la principale revue professionnelle de la discipline, la revue EPS ${ }^{30}$, la sexuation des pratiques reste prégnante car déterminée en amont par une représentation d'aptitudes différentielles repérées chez les élèves. Même parmi les voix les plus réformatrices de l'époque, comme les Comités d'action lycéens, la différenciation entre le corps masculin et le corps féminin doit être lisible «en particulier dans leurs propositions pour l'éducation physique où se distinguent activités des filles et des garçons $»^{31}$.

Fidèle à la philosophie qui anime les réformes scolaires, l'EPS devient une « gare de triage $»^{32}$ des futurs pratiquants non en fonction de l'origine sociale mais en fonction de leur sexe. Cet enseignement prépare les consciences à intégrer les normes collectives en distinguant les pratiques en fonction d'aptitudes attachées respectivement et non indistinctement aux filles et aux garçons :

[...] les sports de combat pour les garçons, les différentes formes de danse pour les jeunes filles, peuvent également contribuer à cultiver chez les jeunes le sens des relations avec autrui ${ }^{33}$.

Le choix des activités est donc conditionné par les normes sociales et par les représentations genrées des rapports humains. Au côté de la combinaison des objectifs associés aux moyens et à l'analyse des intentions éducatives qui constitue la programmation, le choix des activités est différencié selon le sexe. La "gymnastique non sportive» et la « danse et autre expression corporelle» sont en priorité réservées

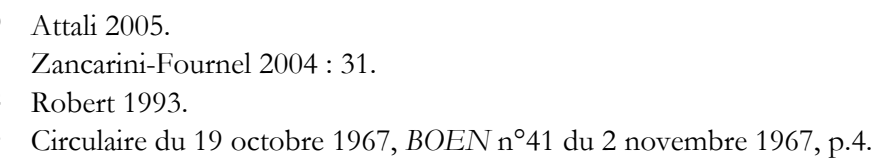


aux filles. Produit et conséquence d'une introduction du sport opérée au même moment qu'un accroissement de la scolarisation, l'enseignement de l'EPS s'appuie ainsi sur deux cultures physiques de référence en même temps qu'il les renforce. Il contribue à développer des formes de sociabilité particulière en fonction des sexes, avec pour conséquence une difficulté évidente de pratiquer ensemble. Dans les rares cas où cela est mis en place, la mixité fait apparaitre de fortes disparités et reste le plus souvent justifiée par des finalités sociales et non plus éducatives.

Alors que la mixité constitue la modalité privilégiée dans la plupart des enseignements, il n'en est pas de même concernant l'EPS: de puissantes précautions demeurent et paraissent d'autant plus indiscutables que les fervents défenseurs de la coéducation comme E. Breuse justifient à leur tour cette gestion sexuée des élèves. Selon ce dernier, les avantages de la coéducation apparaissent en effet incompatibles avec l'utilisation d'une pédagogie exclusivement compétitive; cette dernière se révélant presque toujours dommageable pour le sexe féminin car exclusivement basée sur le principe de la rivalité. «L'éducateur avisé évitera, sous prétexte d'émulation, de créer et d'attiser des sentiments d'opposition entre les garçons et les filles. Les relations entre ces derniers ne devront jamais être placées sur le plan d'une supériorité ou infériorité quelconques ${ }^{34}$. La non mixité en EPS se voit ainsi toujours justifiée.

\section{La mixité : une mascarade égalisatrice !}

À partir du VI e plan (1971-1975), l'objectif d'égalisation des chances se substitue à celui d'égalité des chances. Il devient le thème central des débats éducatifs de la revue EN et justifie une politique éducative fondée sur l'adaptation des contenus aux besoins de chacun:

«Égaliser les chances », ce n'est pas imposer à tous le même moule ; c'est permettre à chacun l'épanouissement d'une personnalité spécifique, et celle-ci ne peut se construire, à partir du patrimoine biologique et

34 Breuse $1970: 128$. 
génétique de chaque individu et compte tenu de son environnement social, que par une éducation scolaire individualisée ${ }^{35}$.

L'école doit compenser les handicaps. Elle doit pouvoir s'adresser à tous les élèves tout en intégrant les nécessités sociales et professionnelles de la Nation, les aspirations culturelles de la jeunesse et les avancées scientifiques et pédagogiques de son temps. Perçue comme inadaptée, elle doit se réformer. Ce renouveau du principe égalisateur permet ainsi de justifier de nouvelles modalités d'enseignement en réactualisant les vertus de la différenciation pédagogique, ce qu'E. Breuse, en 1970, nommait déjà la personnalisation de l'apprentissage. L'individualisation des besoins est alors associée aux déterminismes biologiques et sociaux par l'intermédiaire des notions d'aptitude, de goûts et de motivations. Même si elle est marquée par certaines évolutions, la rhétorique est proche de celle utilisée lors de la réforme Berthoin. Parce que pourvus d'aptitudes différentes, tous les élèves ne nécessitent pas la même pédagogie :

Une véritable égalité des chances de développement intellectuel entre des enfants inégaux exige un enseignement lui-même inégal selon les individus adapté à chaque cas pour être efficacement compensateur ${ }^{36}$.

Dès lors, les groupes de niveaux, associés à une pédagogie du soutien, deviennent le dispositif privilégié pour mettre en œuvre une égalisation qui enregistre davantage les différences qu'elle ne les réduit. Les déterminismes biologiques et socioculturels promeuvent une inégalité en contradiction avec la plupart des discours sur l'école de cette période :

Les voies d'accès à cette formation de base doivent être différenciées selon l'aptitude des élèves et la forme de leur intelligence. Offrir le même enseignement à tous aboutirait en pratique à des résultats contraires à la démocratisation ${ }^{37}$.

35 Cros $1974: 9$.

36 Cros $1974: 9$.

37 Fontanet $1973:$ III. 
Malgré l'intérêt porté à l'égalisation des chances, la mixité n'est abordée qu'une seule fois pour proclamer le rôle de la mixité dans la construction d'une égalité totale entre les hommes et les femmes:

La mixité [...] est un des facteurs essentiels pour supprimer tout esprit de ségrégation entre sexes, toutes les habitudes qui tendent à enfermer les femmes dans certaines tâches bien précises ${ }^{38}$.

L'état de l'opinion et les évolutions sociales initiées par les féminismes de la deuxième vague laisseraient pourtant penser que la discussion sur la mixité est désormais possible. Mais la mixité est déterminée par des évidences que nul ne souhaite interroger. En effet, lorsqu'il est fait référence à des données biologiques, le.la lecteur.trice y associe les différences les plus évidentes dans ce domaine. Or, à cette époque, nul ne conteste que filles et garçons soient par nature inégaux et finalement le discours devient subliminal. Ainsi, l'égalisation des chances ne conduit pas à une appropriation égalitaire des savoirs, comportements ou valeurs par des voies différenciées mais bien à leur différenciation en fonction des critères du genre. La sémantique employée est révélatrice d'un ordre établi où le naturalisme, sans être évoqué, marque toujours de son empreinte les conceptions éducatives structurant la prochaine réforme de l'enseignement. Ainsi Pierre Massé, à l'occasion du colloque national sur l'éducation de janvier 1974, rappelle la nature ségrégative des enseignements comme une évidence, sans qu'il soit nécessaire de mentionner le cas de la différence entre les sexes :

On ne peut oublier qu'il existe au départ des différences d'ordre génétiques qui assignent des limites aux possibilités d'actions ultérieures. Il n'en est que plus impérieux de ne pas ajouter à ces différences innées des inégalités provenant de l'environnement physique et du milieu socioculturel $^{39}$.

Alfred Sauvy confirme ce constat et souligne que «les inégalités de caractère social paraissent toujours plus choquantes que les inégalités

Anon. 1973 : V.

39 Massé 1974. 
naturelles et qu'elles semblent aussi plus faciles à corriger $»^{40}$. L'école est ainsi le lieu de la reproduction d'un conservatisme social et sexué qui n'est pas remis en cause malgré le renouvellement des concepts et l'exercice quotidien de la mixité. Le projet de loi sur la réforme de l'enseignement du $2^{\text {nd }}$ degré, adopté au conseil des ministres du 27 mars 1974, entérine ces orientations qui doivent marquer durablement le système éducatif français :

La première mission de l'école est bien sûr l'accès au savoir, savoir apprendre et savoir être [...] Une seconde mission qui est inséparable de la première est la contribution à l'égalisation des chances. [...]. Cette recherche de l'égalisation des chances ne veut pas dire non plus que tous les élèves doivent recevoir le même enseignement professé de la même manière. Une pédagogie différenciée est seule à même d'aider les plus faibles, sans gêner les plus forts.

Le cadre conceptuel étant posé, les structures et les disciplines doivent permettre l'application pratique. En EPS, les remises en cause fondamentales du début des années $1970^{41}$ accroissent une tendance qui fait de l'EPS autant une discipline éducative qu'un enseignement sportif. Les pratiques qui lui sont associées suscitent un intérêt important chez ceux qui voient en l'école un vecteur de diffusion d'une pratique inégalement répartie. Bien que l'ensemble des enquêtes statistiques $^{42}$ fasse apparaitre un fort déséquilibre entre pratiques féminines et pratiques masculines, les rédacteurs de la revue EN promeuvent un sport pour tous qui, en apparence, refuse de distinguer les deux sexes afin de sérier les efforts à engager. Georges Belbenoit, inspecteur général de l'Éducation nationale, président de l'UFOLEP$\mathrm{USEP}^{43}$, insiste «sur le rôle prépondérant de l'école, où se joue l'avenir du sport pour tous en même temps qu'une part notable de son présent (car il ne faut pas plus assimiler le sport pour tous au sport des femmes

40 Sauvy $1974: 32$.

41 Terret 2000 ; Attali \& Saint-Martin 2004.

42 Attali 2007.

43 Union Française des Euvres Laïques de l'Éducation Physique. Union Sportive de l’Enseignement du Premier degré. 
et des vieillards que l'éducation permanente à la formation continue des adultes) $»^{44}$.

À cette période, le sexe et l'âge continuent de représenter les indicateurs objectifs du maintien d'une forte ségrégation malgré la volonté officielle de faire accéder l'ensemble de la population à une pratique sportive. Isolée et refusant de s'attacher à développer des stratégies pour permettre à tous d'accéder à un savoir identique, l'EPS conserve une posture proche de la période précédente. Ici, l'application du principe d'égalisation des chances prend une forme particulière avec la présence des groupes de niveaux qui constituent un élément différenciateur majeur. Alors qu'ailleurs, la répartition des élèves dépend du niveau de chacun ${ }^{45}$ (aptitude, goûts, motivations ou besoins), les groupes de niveaux en EPS sont d'abord et quasi systématiquement sexués. S'il parait inconcevable de faire pratiquer du rugby à des filles ou de la danse à des garçons, il reste que des activités comme l'athlétisme ou la natation conduisent, par leur caractère performatif, à séparer deux catégories d'élèves dont on imagine difficilement qu'elles puissent se côtoyer en raison de l'écart objectivé qui les sépare. L'évaluation des épreuves sportives s'appuie toujours sur des barèmes de cotation établis notamment par Jean Letessier en 1957, qui en se centrant sur la performance érige l'inégalité des capacités physiques des filles et des garçons en postulat:

Il est d'une importance primordiale que nos épreuves distinguent nettement, comme le sens commun, les différentes valeurs individuelles, les âges, les sexes $[\ldots]^{46}$.

Dans ces conditions, la mesure des apprentissages scolaires reste secondaire; la priorité est donnée à la perception des différences interindividuelles amenant à discriminer les individus et les corps. La différenciation pédagogique, qui devient dans les premières années 1970 un outil de l'égalisation des chances, conduit ainsi en EPS à organiser et à justifier une non mixité sur la base d'une argumentation

44 Belbenoit $1974: 26$.

45 Niveau dont on connaît par ailleurs la dépendance aux déterminismes sociaux.

46 Letessier 1963. 
naturaliste dont cette discipline a tant de difficultés à se départir. L'EPS est donc l'une des rares disciplines à perpétuer explicitement une organisation et des contenus strictement différenciés entre les sexes.

À ce titre, la non-mixité en EPS s'impose donc comme l'expression de l'égalité entre les sexes. L'EPS traduit les limites d'un principe mobilisateur en faisant apparaitre les profondes inégalités derrière les principes égalitaires.

Entre 1959 et 1975 et malgré de généreux discours autour de l'égalité des chances, la mixité n'est jamais explicitement formulée comme étant un objectif prioritaire de la démocratisation de l'institution scolaire française. En France, contrairement aux Pays-Bas ou aux États-Unis, qui font de la coéducation un enjeu éducatif de premier ordre, la mixité intéresse peu malgré les limites et les critiques qu'elle entraîne, surtout en EPS. Entre 1959 et 1975, le mode implicite domine et tout est fait pour convaincre les lecteurs.trices d'une mixité inéquitable en raison de lois naturelles appliquées au domaine social que nul ne peut balayer. Dans ces conditions, les résistances à l'égard de la mixité manifestent la permanence d'une vision inégalitaire des sexes. Cette vision est justifiée par des aptitudes innées différenciées entre les hommes et les femmes. Dans un premier temps, les acteurs du système éducatif s'opposent à cette nouvelle organisation en en dévoilant implicitement les limites et les incohérences. Dans un deuxième temps, ils tentent de s'y adapter en réaffirmant la nécessité d'une différenciation de la pédagogie.

En EPS, cette vision prend une résonance particulière dans la mesure où le corps est le signifiant le plus visible des différences entre les sexes. Pour l'essentiel des acteurs du système éducatif, le corps, conçu comme un véritable outil des apprentissages, est, irrémédiablement et principalement le lieu du déterminisme biologique. La non-mixité et la différenciation sexuée des contenus demeurent la règle fondamentale. Dans les enseignements d'EPS, celle-ci devient une réalité quotidienne avec la présence incontournable des groupes de niveaux. Cette réalité, souvent interprétée comme l'expression des résistances du monde sportif est en fait le résultat des injonctions 
scolaires à l'égard de l'EPS. D'une part, ce choix illustre les résistances de l'école vis-à-vis d'une rencontre physique des corps mais il opérationnalise aussi la conviction, unanimement partagée, d'une différenciation affective, physique et sociale entre les sexes. Enfin, ce choix est le résultat des nécessités égalitaires de l'école dont l'objet est d'instaurer une éducation respectueuse de la spécificité et de la complémentarité des sexes en refusant le principe de leur hiérarchisation, ce qui est en parfaite contradiction avec l'essence de la culture sportive qui sert de référence à l'enseignement de l'EPS, principalement au cours des années 1960. Tout cela se traduit, en France comme en Allemagne, en Angleterre ou aux Pays-Bas, par une impossibilité à questionner les enjeux de la mixité en EPS avant les années 1980. Dans tous ces pays, la mixité en EPS s'établit après celle de l'enseignement général. Ce temps de latence traduit en définitive l'incapacité des sociétés occidentales à penser le corps autrement que sexué et l'incapacité à penser l'égalité au-delà des différences corporelles. Alors que beaucoup d'enseignants et parents restent réticents à une organisation mixte en étant toutefois contraints de la mettre en œuvre, en EPS, les enseignant.e.s perpétuent une organisation et des contenus genrés, ainsi cohérents avec l'ironie des injonctions « égalitaristes » de l'école.

\section{Sources}

ADLER Astrid, 1960, «Y a-t-il un problème avec la jeunesse ?», EN, 4, p. 20.

Anonyme, 1973, "L'éducation nationale et la formation professionnelle féminine », EN, 193, p. V-VIII.

BASTIN Guillaume, 1966, «Enquête sur l'école mixte », Enfance, 4-5, p. 25-43.

BeLbenoit Georges, 1974, « Pour un olympisme quotidien », EN, 195, p. 24-28.

BOUZINAC Roger, 1960, «Rome. Unique objet de nos ressentiments », EN, 24, p. 1617.

BREUSE Edouard, 1970, La coéducation dans les établissements mixtes, Paris, Presses universitaires de France.

BRunsCHWIG Odette-Georges, 1961, «L'éducation mixte », EN, 30, p. 5-7 et p. 10.

CHAussonnet Colette, 1960, «L'éducation physique », EN, 18, p. 10-11.

CHÂTEAU Jean, 1960, «Les aptitudes particulières », EN, 22, p. 1-4.

Courtors Claude, 1967, Co-éducation et mixité, Paris, Le nœud de Carrick et l'action scolaire.

Cros Louis, 1974, «Une école unifiée mais diversifiée », EN, 201, p. 8-9. 
DALE Reginald R., 1965, «Co-education: the verdict of experience. II. A qualitative approach », British Journal of Educational Psychology, 35, 2.

ENCAuSSE Philippe, 1962, Sport et santé. Précis de médecine sportive, Paris, JB Baillière et fils.

FERRAN Pierre, 1970, « De la mixité à la coéducation », EN, 84, p. 21-22.

FERRY Gilles, 1962, «L'éducation physique conserve ses lettres de noblesse », EN, 28, p. 1.

FONTANET Joseph, 1973, «Du rapport Joxe à la loi d'orientation du second degré », EN, 185, p. I-IV.

GUILHEM Jean, 1964, «Une expérience de gymnastique volontaire, EN, 8, p. 8-10.

Herzog Maurice, 1963, «Allocutions de Maurice Herzog», EN, 1, p. 2.

LE LAY Henri, 1965, « Présentation de l'ouvrage de Jeanne Burniaux, L'éducation des jeunes filles », EN, 26, p. 4.

LETESSIER Jean, 1963, « Le certificat de capacité athlétique », Revue EPS, 64, p. 28.

Ministère de l'Éducation Nationale, 1965, «La coéducation», Les cabiers de l'INAS, Journée d'étude franco-belge.

MASSE Pierre, 1974, «Le colloque national sur l'éducation. Réflexions finales », EN, 196, p. IV.

MAUCO Georges, 1969, « La grande peur des adultes », EN, 29, p. 7-9.

SAUVY Alfred, «Demain chances égales ? EN, 21 mars 1974, p. 32

\section{Bibliographie}

AtTALi Michaël \& Jean SAINT-MARTIN, 2004, L'éducation physique depuis 1945. Les étapes d'une démocratisation, Paris, A. Colin.

—, 2005, «Les oubliées de la démocratisation scolaire de l'éducation physique française (1945-2003) ", in Jean SAINT-MArTIN, Thierry TerRET (dir.), Sport et Genre, Apprentissage du genre et institutions éducatives, vol. 3, Paris, L'Harmattan, p. 207226.

ATTALI Michaël, 2005, «Le genre dans la revue EPS», in Jean SAINT-MARTIN, Thierry TERRET (dir.), Sport et Genre..., op. cit., p. 283-302.

—, 2007, «L'explosion des pratiques sportives: massification, diversification, différenciation ", in Philippe TETART, Histoire du sport en France, De la Libération à nos jours, Paris, Vuibert, p. 63-106.

LELIEVRE Françoise \& LELIEVRE Claude, 1991, Histoire de la scolarisation des filles, Paris, Nathan.

Prost Antoine, 1981, Histoire générale de l'enseignement et de l'éducation en France, Paris, Nouvelle Librairie de France.

ROBERT André, 1993, Système éducatif et réformes, Paris, Nathan.

Rogers Rebecca (dir.), 2004, La mixité dans l'éducation, Enjeux passés et présents, Lyon, ENS Éditions.

TERRET Thierry, 2000, Éducation Physique, Sport et Loisir, 1970-2000, Paris, AFRAPS.

Terret Thierry et al., 2005, Sport et Genre, 4 tomes, Paris, L'Harmattan.

ThéBAud Françoise \& Michelle ZANCARINI-FOURnel (dir.), 2003, «Coéducation et mixité », Clio. Histoire, Femmes et Sociétés, 18.

ZANCARINI-FOURNEL Michelle, 2004, «Coéducation, gémination, co-instruction, mixité : débats dans l'Éducation nationale (1882-1976) », in Rebecca ROGERS (dir.), La mixité dans l'éducation, Enjeux passés et présents, Lyon, ENS Éditions, p. 25-34. 